\title{
CD166 promotes the cancer stem-like properties of primary epithelial ovarian cancer cells
}

\author{
Dae Kyoung Kim ${ }^{1}$, Min Hee Ham ${ }^{1}$, Seo Yul Lee ${ }^{1}$, Min Joo Shin ${ }^{1}$, Ye Eun Kim ${ }^{1}$, Parkyong Song ${ }^{2}$, Dong-Soo Suh ${ }^{3} \mathcal{E}$ \\ Jae Ho Kim ${ }^{1,4}$ \\ ${ }^{1}$ Departments of Physiology, ${ }^{2}$ Convergence Medicine, ${ }^{3}$ Obstetrics and Gynecology, School of Medicine, Pusan National University, Yangsan \\ 50612, ${ }^{4}$ Research Institute of Convergence Biomedical Science and Technology, Pusan National University Yangsan Hospital, Yangsan \\ 50612, Korea
}

Cancer stem cells (CSCs) or tumor-initiating cells are thought to play critical roles in tumorigenesis, metastasis, drug resistance, and tumor recurrence. For the diagnosis and targeted therapy of CSCs, the molecular identity of biomarkers or therapeutic targets for CSCs needs to be clarified. In this study, we identified CD166 as a novel marker expressed in the sphereforming CSC population of A2780 epithelial ovarian cancer cells and primary ovarian cancer cells. The $\mathrm{CD} 166^{+}$cells isolated from A2780 cells and primary ovarian cancer cells highly expressed CSC markers, including ALDH1a1, OCT4, and SOX2, and $\mathrm{ABC}$ transporters, which are implicated in the drug resistance of CSCs. The CD166 ${ }^{+}$cells exhibited enhanced CSC-like properties, such as increased sphere-forming ability, cell migration and adhesion abilities, resistance to conventional anticancer drugs, and high tumorigenic potential in a xenograft mouse model. Knockdown of CD166 expression in the sphereforming ovarian CSCs abrogated their CSC-like properties. Moreover, silencing of CD166 expression in the sphere-forming CSCs suppressed the phosphorylation of focal adhesion kinase, paxillin, and SRC. These results suggest that CD166 plays a key role in the regulation of CSC-like properties and focal adhesion kinase signaling in ovarian cancer. [BMB Reports 2020; 53(12): 622-627]

\section{INTRODUCTION}

Epithelial ovarian cancer (EOC) is a common gynecologic cancer with the highest death rate, and it is frequently resistant to conventional cancer therapies (1). The major cause of the poor prognosis of ovarian cancer is the presence of cancer

*Corresponding author. Tel: +82-51-510-8073; Fax: +82-51-5108076; E-mail: jhkimst@pusan.ac.kr

https://doi.org/10.5483/BMBRep.2020.53.12.102

Received 13 May 2020, Revised 2 June 2020, Accepted 8 June 2020

Keywords: ALCAM, Cancer stem cells, CD166, Chemoresistance, Ovarian cancer stem cells (CSCs), a heterogeneous cancer-cell population (2, 3). Improving the therapeutic efficacy of conventional cancer therapy requires identifying diagnostic and/or therapeutic markers that target CSCs. The markers of CSCs that have been identified in ovarian cancer include aldehyde dehydrogenase-1A1 (ALDH1A1), CD133, CD44, and CD117 (4). These proteins are widely accepted as surface markers associated with CSCs. However, the intra-tumor heterogeneity and absence of suitable treatment for complete remission require finding novel CSC markers.

CD166, known as activated leukocyte cell adhesion molecule (ALCAM), is a 105-kDa type I transmembrane glycoprotein of the immunoglobulin superfamily (5). It is expressed predominantly at the cell membrane (6). CD166 has been reported to be increased in various cancers, such as those of the thyroid, head and neck, lungs, and liver (7). The expression of CD166 has been associated with the metastatic capacity of prostate cancer, the invasiveness of cholangiocarcinoma, and the evasion of apoptosis in breast cancer (8-10). The CD166-positive cells have displayed superior sphere-forming ability and cancerinitiating potential in prostate cancer and head and neck squamous-cell carcinoma, respectively $(11,12)$. These reports suggest that CD166 is a marker for CSC-like cells; however, it is still unknown whether CD166 is associated with the CSClike ovarian-cancer phenotypes.

CD166 has five extracellular immunoglobulin-like domains composed of two amino-terminal variable domains, three membrane proximal constant domains, one transmembrane region, and a short, carboxy-terminal cytoplasmic tail (13). These immunoglobulin-like domains mediate homophilic CD166-CD166 interactions (14), which play an essential role in melanoma cell clustering (15). Although CD166 lacks direct actin-binding sites, it is anchored to the actin cytoskeleton by association with the scaffolding proteins syntenin-1 and ezrin via its cytoplasmic tail (16). Through connection with the cytoskeleton, CD166 is likely to be linked to intracellular signaling networks. In liver cancer cells, CD166 has been reported to play a pro-carcinogenic role in liver cancer cells via promoting expression and activation of AKT and Yes-associated protein, a coactivator of Hippo signaling $(17,18)$. Moreover, CD166

ISSN: 1976-670X (electronic edition)

Copyright (C) 2020 by the The Korean Society for Biochemistry and Molecular Biology

(c) This is an open-access article distributed under the terms of the Creative Commons Attribution Non-Commercial License (http://creativecommons.org/licenses/by-nc/4.0) which permits unrestricted non-commercial use, distribution, and reproduction in any medium, provided the original work is properly cited. 
promoted the growth of oral squamous-cell carcinoma by stimulating epidermal growth factor receptor activation (19). These results suggest that CD166 plays a role in the regulation of CSC-like properties by activating pro- tumorigenic signaling pathways; however, the role of CD166 in the pro-tumorigenic signaling in ovarian CSCs has not been explored.

In this study, we identified CD166 as a biomarker and therapeutic target for ovarian CSCs. We explored the role of CD166 in the regulation of CSC-like properties by silencing of CD166 expression in A2780 ovarian cancer cells and primary ovarian cancer cells derived from patients.

\section{RESULTS}

\section{CD166 expression is upregulated in sphere-forming population of $\mathbf{A} 2780$ epithelial ovarian cancer cells} We have previously reported isolation of the sphere-forming CSCs from several epithelial ovarian cancer-cell lines and patient-derived primary EOC-cell lines (20-22). To explore whether CD166 is a potential CSC marker in ovarian cancer, we compared the expression of CD166 on the parental A2780 cells and the A2780-derived sphere-forming cells (A2780-SP). Flow cytometry results indicated that the percentage of CD166expressing cells in A2780-SP cells was $99 \pm 1 \%$, whereas that in A2780 cells was $28 \pm 7 \%$ (Fig. 1A). RT-PCR analysis showed that the mRNA levels of ALDH1a1, a CSC marker, and CD166 in A2780-SP cells were greater than those in A2780 cells (Fig. 1B).
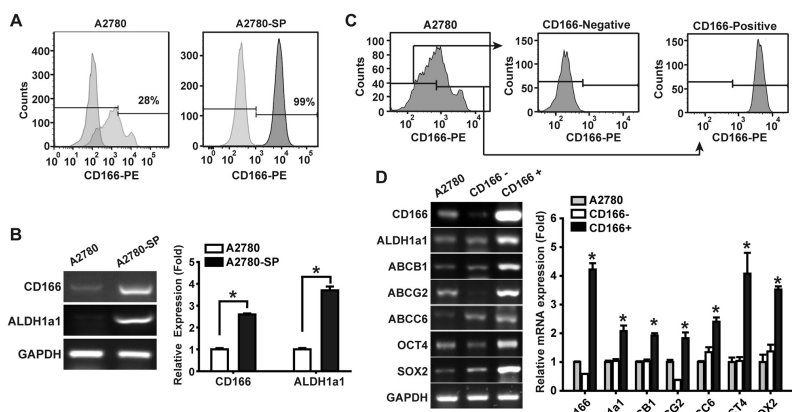

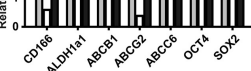

Fig. 1. Increased expression of CD166 in the sphere-forming populations of A2780 ovarian cancer cells. (A) Flow cytometry analysis of CD166 expression in A2780 cells and A2780-SP cells. The percentages of $\mathrm{CD} 166^{+}$population are indicated. (B) RT-PCR analysis of CD166, ALDH1a1, and GAPDH in A2780 and A2780-SP cells (left panel). The mRNA levels of CD166 and ALDH1a1 were quantified and normalized to those of GAPDH (right panel). Data are shown as means $\pm \mathrm{SD}(n=4),{ }^{*} \mathrm{P}<0.05$. (C) We sorted the $\mathrm{CD}_{166^{+}}$and CD166 ${ }^{-}$cells from A2780 cells by flow cytometry, then did FACS analysis of the sorted populations. (D) RT-PCR analysis of CD166 and CSC-related genes in unsorted A2780 cells, $\mathrm{CD}_{166^{+}}$, and $\mathrm{CD}_{166^{-}}$cells (left panel). The mRNA levels of each gene were quantified and normalized to those of GAPDH (right panel). Results are presented as means $\pm \operatorname{SD}(n=4), * P<$ 0.05 vs. CD $166^{-}$.
To explore whether CD166 is a marker for ovarian CSCs, the $\mathrm{CD}_{166^{+}}$and $\mathrm{CD} 166^{-}$cells were isolated from A2780 cells by fluorescence-activated cell sorting with an antiCD166-specific antibody. Flow cytometry analysis demonstrated that the $\mathrm{CD}_{166}{ }^{+}$and the $\mathrm{CD} 166^{-}$populations were separated by cell sorting (Fig. 1C). RT-PCR analysis of the sorted $\mathrm{CD}_{166^{+}}$and $\mathrm{CD} 166^{-}$cells indicated that the CD166 ${ }^{+}$ cells expressed high levels of stemness-associated genes, such as OCT4, SOX2, and the CSC marker ALDH1a1. Furthermore, the expression levels of $\mathrm{ABC}$ transporter genes, which are responsible for the drug resistance property of CSCs, were upregulated in $\mathrm{CD}_{166^{+}}$cells (Fig. 1D).

\section{CD166 is a marker of the CSC-like populations of A2780} ovarian cancer cells

To explore the validity of CD166 as a CSC marker, we compared the proliferation and the sphere-forming abilities between $\mathrm{CD}_{166^{+}}$and $\mathrm{CD} 166^{-}$cells using an MTT assay. The proliferation and sphere-forming abilities of $\mathrm{CD} 166^{+}$cells were greater than those of $\mathrm{CD}_{166^{-}}$cells (Fig. 2A and 2B). Furthermore, the migration and adhesion abilities of $\mathrm{CD} 166^{+}$ cells were remarkably greater than those of $\mathrm{CD}_{166^{-}}$cells (Fig.

A
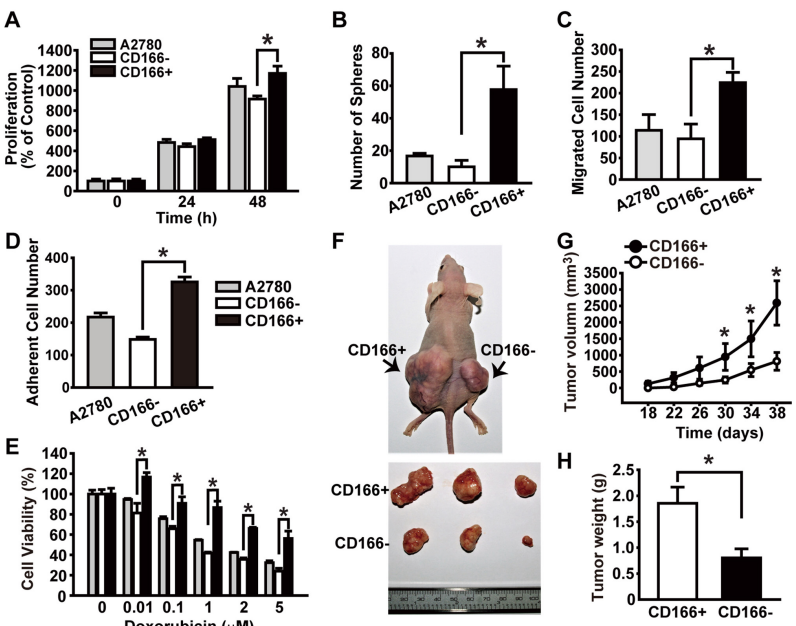

Fig. 2. CD166 is a marker of CSC-like population in A2780 ovarian cancer cells. (A) We measured cell proliferation of unsorted A2780 cells, $\mathrm{CD}_{166^{+}}$, and $\mathrm{CD}_{166^{-}}$cells by MTT assay at the indicated times. (B) We counted sphere numbers of unsorted A2780 cells, $\mathrm{CD}_{166^{+}}$, and $\mathrm{CD} 166^{-}$cells on day 10. Migration (C) and cell adhesion (D) abilities of unsorted A2780 cells, CD166 ${ }^{+}$, and $\mathrm{CD}_{166^{-}}$cells were measured. (E) We treated unsorted A2780 cells, CD166 ${ }^{+}$, and CD166 ${ }^{-}$cells with increasing concentrations of doxorubicin and measured cell viability by MTT at $24 \mathrm{~h}$. (F) Representative image of nude mice (upper image) and tumors (lower image) on day 38 after injection of $\mathrm{CD}_{166^{+}}$and $\mathrm{CD}^{-} 66^{-} \mathrm{A} 2780$ cells. (G) Tumor volumes at the indicated time points after injecting $\mathrm{CD}_{166^{+}}$and $\mathrm{CD}_{166^{-}}$cells were quantified at the indicated time points. $(\mathrm{H})$ Tumor weights on day 38 after injecting CD166 ${ }^{+}$ and $\mathrm{CD}_{166^{-}}$cells were measured. All results are presented as means \pm SD $(n=5), * P<0.05$. 
2C and 2D). We next explored whether CD166 is associated with the drug-resistant characteristic of CSCs. The viability of both $\mathrm{CD}_{166^{+}}$and $\mathrm{CD}_{166^{-}}$cells was decreased by doxorubicin treatment in a dose-dependent manner; however, CD166 ${ }^{+}$ cells exhibited significant resistance to doxorubicin treatment (Fig. 2E).

To assess whether CD166 is associated with the tumorinitiating ability of CSCs in vivo, we measured tumorigenic potentials of the $\mathrm{CD} 166^{+}$and $\mathrm{CD} 166^{-}$cells using a xenograft transplantation model. We subcutaneously injected the CD166 ${ }^{+}$ and the $\mathrm{CD} 166^{-}$cells into the left and right sides of the back of each nude mouse and monitored the in vivo growth of transplanted tumor cells Tumors derived from CD166 ${ }^{+}$cells showed markedly greater tumor growth than did those derived from $\mathrm{CD} 166^{-}$cells (Fig. $2 \mathrm{~F}$ and $2 \mathrm{G}$ ). Furthermore, the xenograft tumors derived from $\mathrm{CD}_{166^{+}}$cells exhibited increased tumor weight compared to those from $\mathrm{CD}_{166}^{-}$cells (Fig. $2 \mathrm{H}$ ). Collectively, these data demonstrated that a $\mathrm{CD} 166^{+}$cell population in A2780 cells displayed CSC-like features; so CD166 can be considered as a marker for ovarian CSCs.

\section{CD166 knockdown abrogates the CSC-like properties of the A2780-SP cells}

To find out the role of CD166 in A2780-SP cells, we knocked down CD166 expression in the cells using siRNA (Fig. 3A). RT-PCR analysis revealed that expression of ALDH1a1, Oct4, Sox2, and $A B C$ transporters (ABCB1, ABCG2, and $A B C C 6$ ) were significantly decreased in response to CD166 knockdown. We next examined the effects of CD166 knockdown on the proliferation, sphere formation, migration, and adhesion of the cells. The proliferative and sphere-forming abilities were attenuated by silencing the expression of CD166 (Fig. 3B and $3 \mathrm{C})$. Furthermore, the migration and adhesion capacity of A2780-SP cells were downregulated by CD166 knockdown (Fig. 3D and 3E). Then, we examined the effect of CD166 knockdown on the drug resistance of A2780-SP cells. In the presence of $1 \mu \mathrm{M}$ doxorubicin, proliferation of A2780-SP cells increased in a time-dependent manner, whereas silencing of CD166 abrogated their proliferation (Fig. 3F). These results suggest that CD166 plays a pivotal role in the proliferation, migration, adhesion, and drug resistance of A2780-SP cells.

To find out whether CD166 is implicated in the activation of intracellular signaling pathways in ovarian CSCs, we examined the effects of CD166 knockdown on the activation of FAK signaling, which plays a key role in the regulation of cell adhesion, proliferation, migration, and survival (23). The siRNA-mediated knockdown of CD166 expression in A2780-SP cells suppressed the phosphorylation of FAK at tyrosine 925, which is known to induce focal adhesion (Fig. 3G) (24). Furthermore, the phosphorylation of downstream signaling molecules of FAK, including paxillin and SRC, was inhibited by the CD166 knockdown.

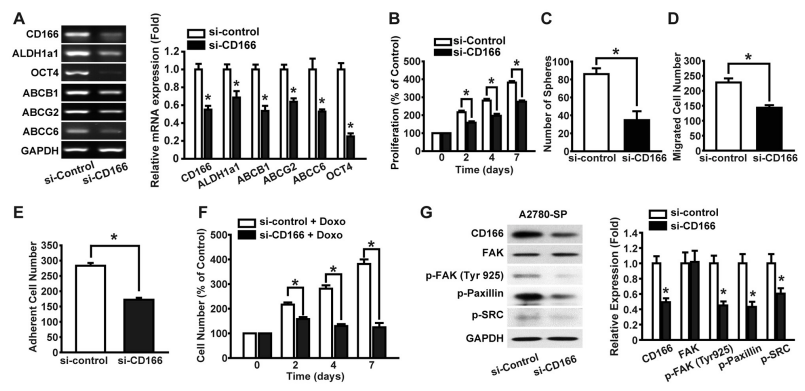

Fig. 3. Effects of CD166 knockdown on the CSC-like characteristics of A2780-SP cells. (A) Expression of CSC-related genes in CD166 siRNA- and control siRNA-transfected A2780-SP cells. We measured the mRNA levels of CD166 and CSC-related genes in control siRNA (si-control)- or CD166 siRNA (si-CD166)-transfected A2780-SP cells by RT-PCR analysis (left panel); the mRNA levels of each gene were quantified and normalized to those of GAPDH (right panel). Cell proliferation (B) and sphere-forming abilities (C) of si-control- or si-CD166-transfected A2780-SP cells were measured until day 7. Migration (D) and cell adhesion (E) abilities of si-control- or si-CD166-transfected A2780-SP cells were measured. (F) Drug resistance of si-control- or si-CD166-transfected A2780-SP cells under treatment of doxorubicin $1 \mu \mathrm{M}$ was assessed for 7 days. (G) Effects of CD166 knockdown on the FAKassociated signaling pathway activation. A2780-SP cells were transfected with either si-control or si-CD166. We measured the expression and phosphorylation levels of FAK, paxillin, and SRC by Western blotting at $48 \mathrm{~h}$ after siRNA transfection (left panel). The band intensities were quantified and normalized to those of $\mathrm{GAPDH}$ (right panel). Results are presented as means \pm SD $(n=$ 4), $* P<0.05$ vs. si-control.

\section{CD166 knockdown abrogates the CSC-like properties of primary ovarian CSCs}

We have reported the isolation and cultivation of sphereforming primary CSCs from tumor tissues of patients with EOC $(20,25)$. In this study, we used a sphere-forming primary EOC cell line, EOC12-SP, which has been previously established (20). FACS analysis with the PE-labeled CD166 antibody showed that CD166 is highly expressed in the EOC12-SP cells (Fig. 4A). To find out whether CD166 regulates stemness in primary ovarian CSCs, we silenced CD166 expression in EOC12-SP cells by transfection with a CD166-specific siRNA. RT-PCR analysis revealed that the mRNA levels of Oct4, Sox2, ALDH1a1, ABCB1, ABCG2, and ABCC6 in EOC12-SP cells was significantly decreased by silencing the CD166 gene (Fig. $4 B)$.

We next explored the role of CD166 in the proliferation, drug resistance, spheroid formation, cell migratory, and adhesive capacities of EOC12-SP cells by silencing the CD166 expression. The proliferation rate, the number of spheroids, cell migration, and adhesion abilities of EOC12-SP cells were remarkably decreased by silencing the CD166 gene (Fig. 4C-F). In the presence of doxorubicin, control siRNA-transfected EOC12-SP cells proliferated in a time-dependent manner; however, proliferation of the CD166-silenced cells was almost 

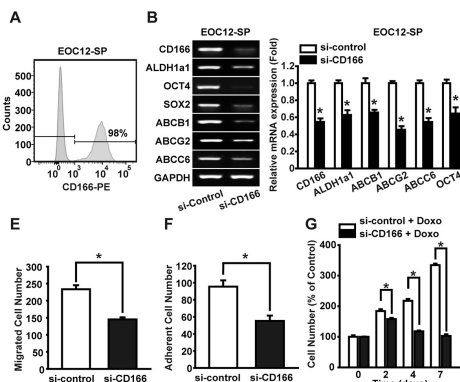

c
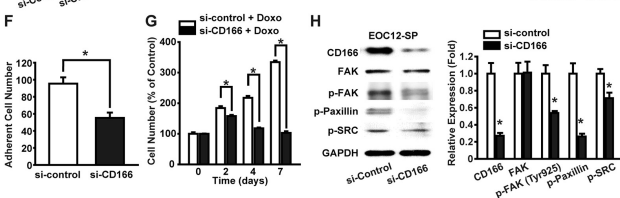

Fig. 4. Effects of CD166 knockdown on the CSC-like characteristics of primary ovarian CSCs. (A) We measured the expression of CD166 in EOC12-SP with flow cytometry analysis. (B) RT-PCR analysis of CSC-related genes in si-control- or si-CD166-transfected EOC12-SP (left panel). The mRNA levels of each gene were quantified and normalized to those of GAPDH (right panel). (C) Cell proliferation of si-control- or si-CD166-transfected EOC12-SP was measured for 7 days. (D) Representative images of spheroids formed from si-control or si-CD166-transfected EOC12-SP cells at day 7 (upper panel) Scale bar $=100 \mu \mathrm{m}$. The numbers of spheroids were quantified at day 7 (lower panel). Cell migration (E) and adhesion (F) abilities of si-control or si-CD166-transfected EOC12-SP. (G) Drug resistance of si-control- or si-CD166-transfected EOC12-SP under doxorubicin treatment was assessed for 7 days. $(\mathrm{H})$ Effects of CD166 knockdown on the FAK-associated signaling pathway activation. EOC12-SP was transfected with either control or CD166 siRNA, and we measured the expression and phosphorylation levels of FAK, paxillin, and SRC by Western blotting at $48 \mathrm{~h}$ after siRNA transfection (left panel), and the band intensities were quantified and normalized to those of GAPDH (right panel). Results are presented as means $\pm \mathrm{SD}(n=4),{ }^{*} \mathrm{P}<0.05$ vs. si-control.

completely inhibited by doxorubicin treatment (Fig. 4G).

Consistent with the results with A2780-SP cells, the phosphorylation levels of $F A K$, paxillin, and SRC were significantly decreased in the CD166-silenced EOC12-SP (Fig. 4H). These results confirm that CD166 plays a key role in regulating the CSC-like properties and the FAK-associated signaling pathway in primary ovarian CSCs.

\section{DISCUSSION}

In this study, we demonstrate that CD166 is a novel marker of CSCs isolated from the A2780 ovarian cancer-cell line or primary EOC. The $\mathrm{CD}_{166}{ }^{+}$cancer cells exhibited increased expression of CSC markers. Furthermore, sphere formation, cell migration, drug resistance, and tumorigenic potential were augmented more in the $\mathrm{CD} 166^{+} \mathrm{A} 2780$ cells than in those in CD166 ${ }^{-}$A2780 cells. In uveal melanoma, the CD166 ${ }^{+}$subpopulation exhibited better migratory capabilities than did the CD166 ${ }^{-}$cells (26). High levels of CD166 expression has been shown to correlate to poor prognosis in various types of cancer (7), and a meta-analysis suggests CD166 as a risk factor for cancers (27). Therefore, it is likely that the expression of CD166 will be highly useful as a CSC marker for predicting the prognosis of patients with ovarian cancer.
We demonstrated that silencing of CD166 expression attenuated the expression of stemness markers, proliferation, migration, adhesion, and drug resistance of A2780-SP cells and primary ovarian CSCs. CD166 has been known to mediate cell-cell cohesion, enabling the formation of tumor aggregates, extravasation, and metastasis (28). CD166 has been reported to promote cell migration, invasion, and metastasis in earlystage endometrioid endometrial cancer, and recurrence-free survival was poorer in patients with CD166-positive rather than CD166-negative tumors (29). Downregulation of CD166 inhibited invasion, migration, and epithelial-mesenchymal transition in human nasopharyngeal-carcinoma cell line (30). Furthermore, silencing of CD166 expression inhibited cell-cell adhesion and tumor growth in a murine model of triple negative breast cancer (31). The immunoglobulin-like domains of CD166 mediate homophilic CD166-CD166 interaction, which plays a key role in the regulation of cell adhesion and migration $(13,14)$. These results suggest that CD166 plays important roles in maintaining the CSC-like properties.

Although CD166 has been implicated in the regulation of cell adhesion, it is still elusive how CD166 controls the CSC-like properties. A possible explanation is that the association of CD166 with actin cytoskeleton and other binding partners may affect intracellular signaling. CD9, a member of the tetraspanin family, has been shown to play a pivotal role in the regulation of CD166-mediated cell adhesion by increasing the homophilic interaction and clustering of CD166 (32). CD9 associates with not only CD166 but also integrin adhesion receptors, which are implicated in the activation of cell adhesion-dependent activation of FAK, suggesting that CD166 may be linked to FAK signaling via interaction with CD9. We showed here that silencing of CD166 abrogated cell adhesioninduced phosphorylation of FAK, paxillin, and SRC in ovarian CSCs. In addition, CD166 has been reported to stimulate activation of epidermal growth factor receptor, AKT, and YAP (17-19). These results suggest that CD166 may regulate intracellular signaling associated with the CSC-like properties through interacting with various signaling molecules.

In summary, this study demonstrates that the cell-adhesion molecule CD166 is a marker of ovarian CSC-like populations in not only cancer cell lines but also in patient-derived primary EOC. It also plays a key role in the regulation of tumorigenic and drug-resistance potentials of CSCs. Therefore, CD166 may be a promising therapeutic target for therapy of various types of tumors, including ovarian cancer.

\section{MATERIALS AND METHODS}

\section{Materials}

We purchased:

- RPMI1640 medium from Welgene.

- HBSS (Hank's Balanced Salt Solution), trypsin, fetal bovine serum (FBS), B-27 Supplement, penicillin, streptomycin, and Accutase cell detachment solution from Life Technol- 
ogies.

- Basic fibroblast growth factor (bFGF) and epidermal growth factor (EGF) from R\&D Systems.

- Cell culture plates for adherent cells from Thermo Fisher Scientific (Waltham, MA).

- Culture plates with ultra-low attachment surface from Corning Life Sciences (Durham, NC) for culture of sphere cells.

- PE-labeled mouse anti-human CD166 antibody (\#559263), 7-AAD (\#559925) and Ig Isotype Control Cocktail-C (\#558 659) from BD Pharmingen.

- Antibodies against glyceraldehyde-3-phosphate dehydrogenase (GAPDH) from EMD Millipore (Billerica, MA).

- Antibodies against CD166 (ab109215) and human recombinant proteins against CD166 (ab215630) from Abcam.

- Antibodies against p-PAXILLIN (2541S), p-SRC (2101S), p-FAK (3284S), and FAK (3285S) from Cell Signaling Technology.

Isolation of sphere-forming CSCs isolated from an A2780 cell line and tumor tissue of ovarian-cancer patients

A human ovarian-cancer cell line A2780 was maintained in the culture medium (RPMI1640 medium supplemented with $10 \% \mathrm{FBS})$. We isolated sphere-forming CSC populations from A2780 ovarian cell line and tumor tissues of ovarian cancer patients and cultured them in a CSC culture medium (Neurobasal media $(1 \times)$ (Life Technologies) supplemented with 20 $\mathrm{ng} / \mathrm{ml} \mathrm{bFGF}, 10 \mathrm{ng} / \mathrm{mL}$ EGF, $2.5 \mu \mathrm{g} / \mathrm{mL}$ amphotericin, 100 $\mathrm{IU} / \mathrm{ml}$ penicillin, $100 \mu \mathrm{g} / \mathrm{ml}$ streptomycin, and B-27 Supplement $(50 \times)$ (Life Technologies, without serum) in Ultra-Low Attachment plates as previously reported (20).

\section{Tumorigenesis assay in a xenograft tumor model}

All animal studies adhered to protocols approved by the Pusan National University Institutional Animal Care and Use Committee. $\mathrm{CD} 166^{+}$and $\mathrm{CD}^{-} 66^{-}$populations of $\mathrm{A} 2780$ cells $(1 \times$ $10^{5}$ cells) were re-suspended in $100 \mu$ lof Matrigel solution (1:1 dilution with RPMI) and injected subcutaneously into the right and left flanks of 6- to 8-week-old female BALB/c-nu/nu mice. We then inspected the mice transplanted with tumor cells biweekly for tumor appearance by means of visual observation and palpation. We measured the length $(\mathrm{mm})$, width $(\mathrm{mm})$, and height $(\mathrm{mm})$ of the tumor masses twice weekly using electronic Vernier calipers and calculated the tumor volumes $\left(\mathrm{mm}^{3}\right)$ as (length $\times$ width $\times$ height)/2. We measured tumorigenicity every 3-4 days beginning at 18 days after A2780 cell injection. All of the mice were sacrificed by anesthetic overdose on day 38 .

\section{Statistical analysis}

The data are presented as the mean \pm S.D. We calculated statistical significance by using two-tailed unpaired Student's $t$-tests. Further detailed methods are available in the Supporting Information.

\section{ACKNOWLEDGEMENTS}

Support for this research was by the MRC programs (NRF2015R1A5A2009656) of the National Research Foundation of Korea funded by the Ministry of Education, Science and Technology (NRF-2016R1D1A1B03935769; NRF-2017R1A2B400 9021). and the Korea Health Technology R\&D Project, Ministry of Health and Welfare (HI17C1635).

\section{CONFLICTS OF INTEREST}

The authors have no conflicting interests.

\section{REFERENCES}

1. Reid BM, Permuth JB and Sellers TA (2017) Epidemiology of ovarian cancer: a review. Cancer Biol Med 14, 9-32

2. Ahmed N, Kadife E, Raza A, Short M, Jubinsky PT and Kannourakis G (2020) Ovarian cancer, cancer stem cells and current treatment strategies: a potential role of magmas in the current treatment methods. Cells 9, 719

3. Flesken-Nikitin A, Hwang Cl, Cheng CY, Michurina TV, Enikolopov G and Nikitin AY (2013) Ovarian surface epithelium at the junction area contains a cancer-prone stem cell niche. Nature 495, 241-245

4. Garson K and Vanderhyden BC (2015) Epithelial ovarian cancer stem cells: underlying complexity of a simple paradigm. Reproduction 149, R59-70

5. Swart GW (2002) Activated leukocyte cell adhesion molecule (CD166/ALCAM): developmental and mechanistic aspects of cell clustering and cell migration. Eur J Cell Biol 81, 313-321

6. Tachezy M, Zander H, Gebauer F et al (2012) Activated leukocyte cell adhesion molecule (CD166)-its prognostic power for colorectal cancer patients. J Surg Res 177, e1520

7. Weidle UH, Eggle D, Klostermann S and Swart GW (2010) ALCAMCD166: cancer-related issues. Cancer Genomics Proteomics 7, 231-243

8. Jezierska A, Matysiak W and Motyl T (2006) ALCAM CD166 protects breast cancer cells against apoptosis and autophagy. Med Sci Monit 12, BR263-273

9. Hansen AG, Arnold SA, Jiang M et al (2014) ALCAM CD166 is a TGF-beta-responsive marker and functional regulator of prostate cancer metastasis to bone. Cancer Res 74, 1404-1415

10. Adisakwattana P, Suwandittakul N, Petmitr S, Wongkham S, Sangvanich P and Reamtong O (2015) ALCAM is a novel cytoplasmic membrane protein in TNF-alpha stimulated invasive cholangiocarcinoma cells. Asian Pac J Cancer Prev 16, 3849-3856

11. Yan $M$, Yang X, Wang $L$ et al (2013) Plasma membrane proteomics of tumor spheres identify CD166 as a novel marker for cancer stem-like cells in head and neck squamous cell carcinoma. Mol Cell Proteomics 12, 32713284

12. Jiao J, Hindoyan A, Wang S et al (2012) Identification of 
CD166 as a surface marker for enriching prostate stem/ progenitor and cancer initiating cells. PLoS One 7, e42564

13. von Lersner A, Droesen L and Zijlstra A (2019) Modulation of cell adhesion and migration through regulation of the immunoglobulin superfamily member ALCAM/CD166. Clin Exp Metastasis 36, 87-95

14. van Kempen LC, Nelissen JM, Degen WG et al (2001) Molecular basis for the homophilic activated leukocyte cell adhesion molecule (ALCAM)-ALCAM interaction. J Biol Chem 276, 25783-25790

15. Swart GW, Lunter PC, Kilsdonk JW and Kempen LC (2005) Activated leukocyte cell adhesion molecule (ALCAW CD166): signaling at the divide of melanoma cell clustering and cell migration? Cancer Metastasis Rev 24, 223-236

16. Tudor C, te Riet J, Eich C et al (2014) Syntenin-1 and ezrin proteins link activated leukocyte cell adhesion molecule to the actin cytoskeleton. J Biol Chem 289, 13445-13460

17. Yu W, Wang J, Ma L et al (2014) CD166 plays a pro-carcinogenic role in liver cancer cells via inhibition of FOXO proteins through AKT. Oncol Rep 32, 677-683

18. Ma L, Wang J, Lin J, Pan Q, Yu Y and Sun F (2014) Cluster of differentiation 166 (CD166) regulated by phosphatidylinositide 3-Kinase (PI3K)/AKT signaling to exert its anti-apoptotic role via yes-associated protein (YAP) in liver cancer. J Biol Chem 289, 6921-6933

19. Jia G, Wang X, Yan M, Chen W and Zhang P (2016) CD166-mediated epidermal growth factor receptor phosphorylation promotes the growth of oral squamous cell carcinoma. Oral Oncol 59, 1-11

20. Seo EJ, Kwon YW, Jang IH et al (2016) Autotaxin regulates maintenance of ovarian cancer stem cells through lysophosphatidic acid-mediated autocrine mechanism. Stem Cells 34, 551-564

21. Choi EJ, Seo EJ, Kim DK et al (2016) FOXP1 functions as an oncogene in promoting cancer stem cell-like characteristics in ovarian cancer cells. Oncotarget 7, 35063519

22. Kang KT, Kwon YW, Kim DK et al (2018) TRRAP stimulates the tumorigenic potential of ovarian cancer stem cells. BMB Rep 51, 514-519
23. Parsons JT (2003) Focal adhesion kinase: the first ten years. J Cell Sci 116, 1409-1416

24. Westhoff MA, Serrels B, Fincham VJ, Frame MC and Carragher NO (2004) SRC-mediated phosphorylation of focal adhesion kinase couples actin and adhesion dynamics to survival signaling. Mol Cell Biol 24, 81138133

25. Seo EJ, Kim DK, Jang IH et al (2016) Hypoxia-NOTCH1SOX2 signaling is important for maintaining cancer stem cells in ovarian cancer. Oncotarget 7, 55624-55638

26. Djirackor L, Kalirai $\mathrm{H}$, Coupland SE and Petrovski G (2019) CD166high uveal melanoma cells represent a subpopulation with enhanced migratory capacity. Invest Ophthalmol Vis Sci 60, 2696-2704

27. Xiao M, Wang X, Yan M and Chen W (2016) A systematic evaluation for the potential translation of CD166-related expression as a cancer biomarker. Expert Rev Mol Diagn $16,925-932$

28. Degen WG, van Kempen LC, Gijzen EG et al (1998) MEMD, a new cell adhesion molecule in metastasizing human melanoma cell lines, is identical to ALCAM (activated leukocyte cell adhesion molecule). Am J Pathol 152, 805813

29. Devis L, Moiola CP, Masia N et al (2017) Activated leukocyte cell adhesion molecule (ALCAM) is a marker of recurrence and promotes cell migration, invasion, and metastasis in early-stage endometrioid endometrial cancer. J Pathol 241, 475-487

30. Sun Y, Lin H, Qu S et al (2019) Downregulation of CD166 inhibits invasion, migration, and EMT in the radio-resistant human nasopharyngeal carcinoma cell line CNE-2R. Cancer Manag Res 11, 3593-3602

31. Ferragut F, Cagnoni AJ, Colombo LL et al (2019) Dual knockdown of Galectin-8 and its glycosylated ligand, the activated leukocyte cell adhesion molecule (ALCAM CD166), synergistically delays in vivo breast cancer growth. Biochim Biophys Acta Mol Cell Res 1866, 13381352

32. Gilsanz A, Sanchez-Martin L, Gutierrez-Lopez MD et al (2013) ALCAM/CD166 adhesive function is regulated by the tetraspanin CD9. Cell Mol Life Sci 70, 475-493 